\title{
THE EFFECT OF HYDROGEN DIFFUSION BEHAVIOR ON THE EMBRITTLEMENT OF STEEL UNDER HYDROGEN ATMOSPHERIC CONDITION
}

\author{
A. Toshimitsu Yokobori Jr, ${ }^{1}$ Yoru Wada, ${ }^{2}$ Tadao Iwadate, ${ }^{2}$ Kunihiro Yamada, ${ }^{3}$ Toshihito Ohmi ${ }^{1}$ \\ ${ }^{1}$ Graduate School of Engineering Dept. of Nanomechanics, Tohoku University, Aoba 01, Aramaki, Aoba-ku, Sendai, \\ \#980-8579, JAPAN \\ ${ }^{2}$ The Japan Steel Works, LTD, 4, Chatsu-mashi ,Muroran, \#051-8505, JAPAN \\ ${ }^{3}$ Dept of Mechanical Engineering, Faculty of Science and Engineering, Keio University, 3-14-1, Hiyoshi, Koohoku-ku, \\ Yokohama \#223-8522, JAPAN
}

\begin{abstract}
Much hydrogen penetrates from a container wall surface when a petroleum-refining reactor operates under high temperature and pressurized hydrogen environmental condition. Major part of the hydrogen remains in the wall for a long time during cooling process when the reactor is out of operation, which results in the inducement of peculiar delayed fracture. In this paper, the proposed analytical method on hydrogen diffusion and concentration around a crack tip was applied to this phenomenon and the mechanism of this behavior was clarified.
\end{abstract}

\section{INTRODUCTION}

Much hydrogen penetrates from a container wall surface,when a petroleum-refining reactor operates under high temperature and pressurized hydrogen environment condition. Major part of the hydrogen remains in the wall for a long time during cooling process when the reactor is out of operation, which results in the inducement of delayed fracture. [1,2]

However the experimental research of such hydrogen embrittlement is technically difficult at the point of the method of enclosing hydrogen in a specimen and maintaining its state during experiment.

Recently, using a large specimen, the test method of hydrogen embrittlement under the state of maintaining the initial residual hydrogen distribution has been proposed. [3]

By adopting this test method, the experimental research of hydrogen embrittlement was conducted for time sequential deteriorated Cr-Mo-V steel in the 1960's (petroleum refining reactor materials). [2,3] The yield stress of this material takes $500 \sim 600 \mathrm{MPa}$. Due to previous theoretical results, [4-6] the value of yield stress was found to exist in the region of not so strong sensitivity of hydrogen embrittlement. Furthermore the experimental results of hydrogen embrittlement for this material show that peculiar fracture behavior occurs depending on applied stress rate. [3]

In this paper, the proposed analytical method on hydrogen diffusion and concentration around a crack tip [4-6] was applied to this phenomenon mentioned above and the mechanism of this peculiar fracture behavior was clarified.

\section{PHYSICAL MODEL AND BASIC EQUATION}

The basic equation of hydrogen diffusion is essentially same as that of previous paper [4-6] and it is written by Eq. (1) with the addition of a stress induced diffusion, [7,8] 
$\frac{\partial C}{\partial t}=D \nabla\left(\alpha \nabla C+\frac{C}{R T} \nabla v\right)$

$v=-\sigma_{p} \Delta V$,

where $C$ is hydrogen concentration, $D$ is diffusion constant ( $D=12.7 \times 10^{-11} \mathrm{~m}^{2} / \mathrm{s}[9,10]$ ), $R$ is universal gas constant $(8.314 \mathrm{Nm} / \mathrm{molK}), T$ is absolute temperature, $t$ is time, $\Delta V$ is partial molar volume of hydrogen in the alloy considered $\left(2 \times 10^{-6} \mathrm{~m}^{3} / \mathrm{mol}\right)[9,10], \sigma_{p}$ is hydrostatic stress.

Local elastic stress field around a crack tip is given by Eq. (2), [11]

$\sigma_{P}=\frac{2}{3}(1+v) \frac{K \cos (\theta / 2)}{\sqrt{2 \pi r}}+\frac{1}{3} \sigma_{0}$,

where $v$ is Poisson's ratio, $\mathrm{K}$ is stress intensity factor, $\sigma_{0}$ is gross stress.

Local plastic stress filed around a crack tip is given by Eq. (3) [4,5] based on Hill's equation, [12]

$\sigma_{P}=\frac{1}{3}(1+v) \sigma_{y s}\left\{1+2 \ln \left(\frac{r}{\rho}\right)\right\} \cos \frac{\theta}{2}$.

The basic equations for hydrogen diffusion and concentration around a crack tip are given by Eqs. (4) and (5) in the elastic and plastic region respectively.

Elastic region

$$
\frac{\partial C}{\partial t}=D\left(\frac{\partial^{2} C}{\partial r^{2}}+\frac{1}{r} \frac{\partial C}{\partial r}+\frac{1}{r^{2}} \frac{\partial^{2} C}{\partial \theta^{2}}\right)+\frac{D K^{*}}{2 R T}\left\{\frac{\cos (\theta / 2)}{r^{3 / 2}} \frac{\partial C}{\partial r}+\frac{\sin (\theta / 2)}{r^{5 / 2}} \frac{\partial C}{\partial \theta}\right\},
$$

where $K^{*}=\frac{\sqrt{2}}{3} \frac{1}{\sqrt{\pi}}(1+v) K \Delta V$.

Plastic region

$$
\frac{\partial C}{\partial t}=D\left(\frac{\partial^{2} C}{\partial r^{2}}+\frac{1}{r} \frac{\partial C}{\partial r}+\frac{1}{r^{2}} \frac{\partial^{2} C}{\partial \theta^{2}}\right)+\frac{D U^{*}}{R T}\left[\frac{\sin (\theta / 2)}{2 r^{2}}\left\{\frac{1}{2}+\ln \left(\frac{r}{\rho}\right)\right\} \frac{\partial C}{\partial \theta}-\frac{\cos (\theta / 2)}{r} \frac{\partial C}{\partial r}\right],
$$

where $U^{*}=\frac{2}{3}(1+v) \sigma_{y s} \Delta V$.

Applied stress increases with increase in the time of stress application during loading process as shown in Eq. (6),

$$
\sigma=\dot{\sigma}_{R} t
$$

where $\dot{\sigma}_{R}$ is the applied loading rate. Correspondingly, the plastic region increases as is shown in Eq. (7),

$r_{Y}=\frac{1}{2 \pi}\left(\frac{K}{\sigma_{Y}}\right)^{2}=\frac{a}{2}\left(\frac{\dot{\sigma} t}{\sigma_{Y}}\right)^{2}$,

where $r_{Y}$ is the distance of elastic - plastic boundary from a crack tip.

The position of elastic plastic boundary was reset at the mesh point of finite difference element corresponding to the increase in $r_{Y}$. The numerical accuracy of the determination of $r_{Y}$ was kept less than $10^{-4}$. Yield stress, $\sigma_{y}$ is given by Eq. (8), [9.10]

$\sigma_{Y}=P_{C} \sigma_{y s}=1.125 \sigma_{y s}$,

where $P_{C}$ is plastic constraint coefficient, $\sigma_{y s}$ is uniaxial tensile yield stress. Physical model is shown in Fig.1. 


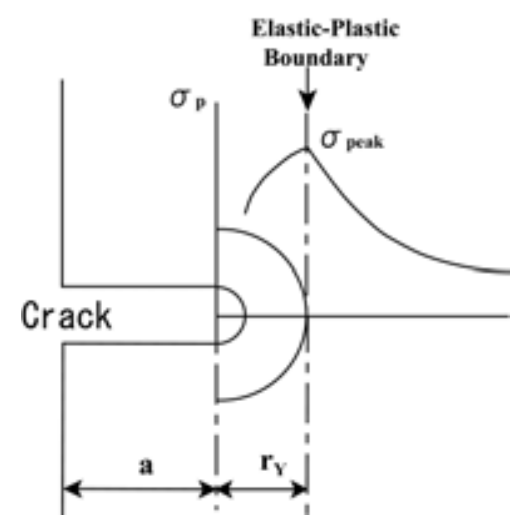

Fig. 1 Physical model of elastic-plastic local stress field around a crack tip

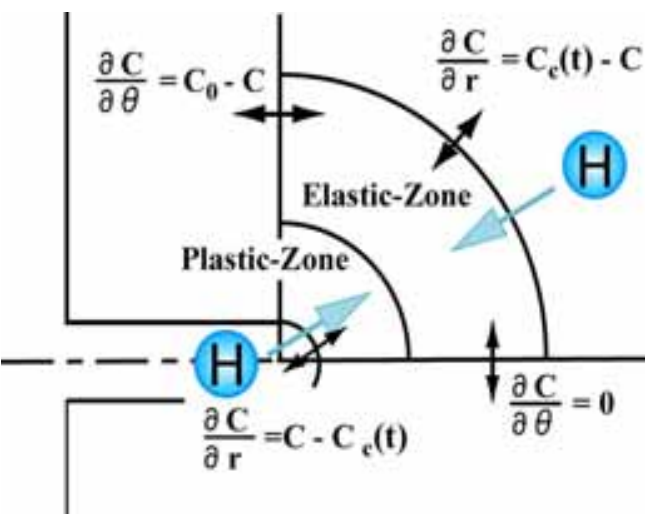

Fig. 2 Physical model of hydrogen penetration

3 RESULTS OF ANALYSES AND COMPARISON WITH EXPERIMENTAL RESULTS

3.1 Hydrogen embrittlement and cracking for a cracked specimen under hydrogen atmospheric and constant stress rate conditions

To simulate the hydrogen atmospheric condition, hydrogen is assumed to penetrate from both of crack surface free boundary and outer boundary as shown in Fig.2.

Eq. (9) is adopted as a function of hydrogen penetration, [5,6]

$C_{c}(t)=\left(C_{c \max }-C_{0}\right)\{1-\exp (-\eta t)\}+C_{0}$,

where $C_{c \max }$ is maximum hydrogen emission concentration in the region of its penetration, $C_{0}$ is initial hydrogen concentration. $\eta$ is a constant.

Using the same material constants as those of petroleum refining reactor, numerical results of hydrogen concentration behaviors obtained by this analysis are shown in Figs 3 and 4 under low and high stress rate conditions respectively.

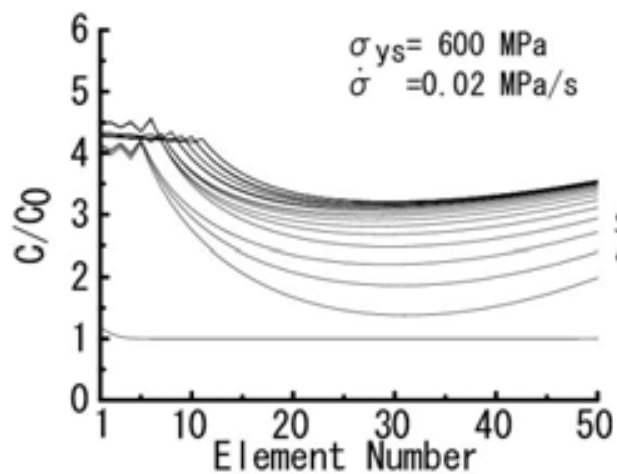

Fig. 3 Hydrogen distribution under low stress rate condition for a cracked specimen

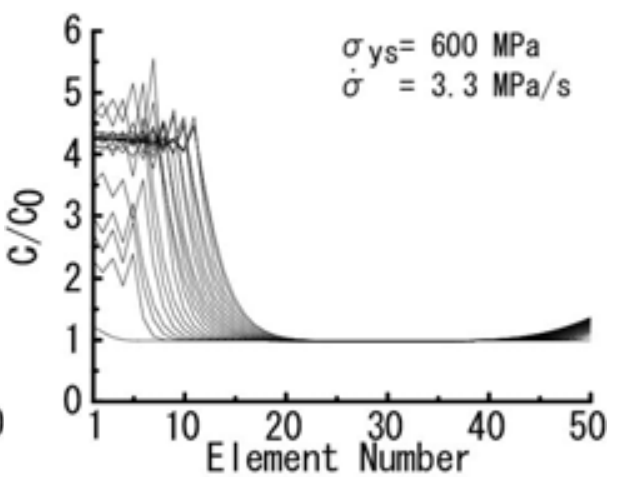

Fig. 4 Hydrogen distribution under high stress rate condition for a cracked specimen

Under low stress rate condition, penetration of hydrogen from outer surface typically occurs and hydrogen distribute through whole region.

On the other hand, under high stress rate condition, hydrogen localizes and concentrates at the elastic plastic boundary. 
The comparison of these numerical results with experimental results of CT specimens for time sequential deteriorated Cr-Mo-V steel in the 1960's [3] was conducted. The experimental results show that under low stress rate condition ( $\dot{K}=0.005 \mathrm{MPa} \sqrt{m} / \mathrm{s}$ ), intergranular cracking takes major part of whole fracture surface and under high stress rate condition ( $\dot{K}=0.05 \mathrm{MPa} \sqrt{\mathrm{m}} / \mathrm{s}$ ), intergranular cracking localizes around a crack tip. [3] Correspondingly to these experimental results, fracture strength under low stress rate condition was found to remarkably decrease as compared with that under high stress rate condition. [3] If these intergranular cracking was considered to be caused by hydrogen embrittlement, these experimental results are in good agreement with the results obtained by numerical analyses of hydrogen diffusion and concentration.

From these results mentioned above, the behavior of hydrogen embrittlement for a cracked specimen was found to be dominated by applied stress rate. The low stress rate application causes typical hydrogen embrittlement due to the wide distribution of hydrogen as shown in Fig.5.

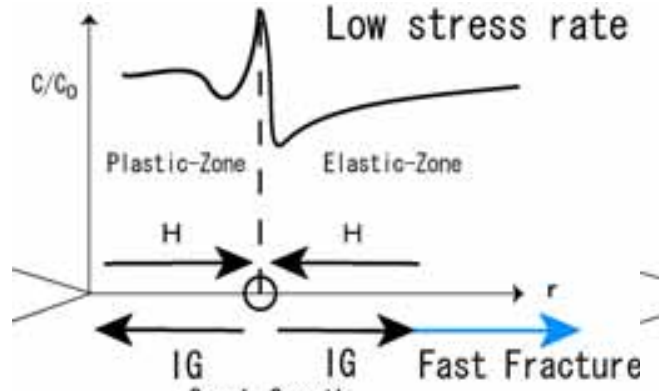

Crack Growth

(a) Acceleration rate crack growth

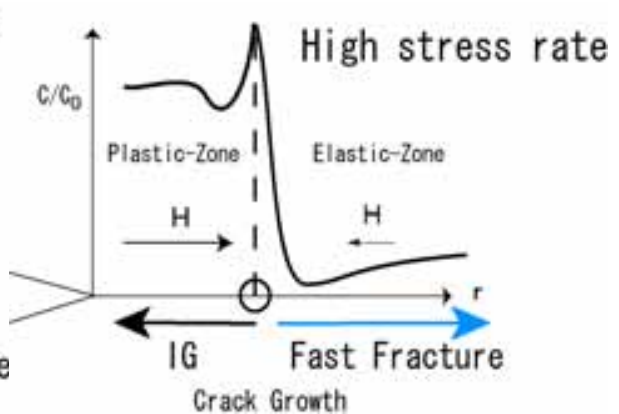

(b) Constant rate crack growth

Fig. 5 Hydrogen embrittlement mechanism under high and low stress rate condition

3.2 Hydrogen embrittlement and cracking for a smooth specimen under hydrogen atmospheric and constant stress rate conditions

For a smooth specimen, hydrogen embrittlement cracking was considered to be originated from a trigger point in the material. [13] Therefore, in this paper, as the physical model of numerical analysis, a pre-existent crack was adopted corresponding to a trigger point and hydrogen was assumed to penetrate from outer surface as shown in Fig.6.

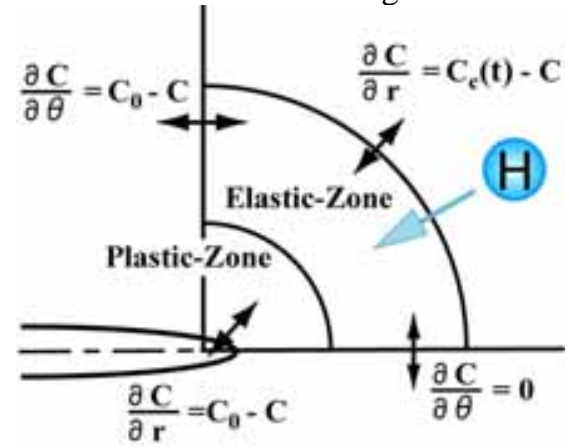

Fig. 6 Physical model of hydrogen penetration toward a pre existent crack for a smooth specimen 
Numerical results were shown in Figs.7 and 8. Under low stress rate condition, hydrogen, which penetrates from outer surface, at first diffuses to the vicinity of a crack tip. The tip of hydrogen distribution goes back, corresponding to the increase in elastic - plastic boundary due to the increase in applied stress.

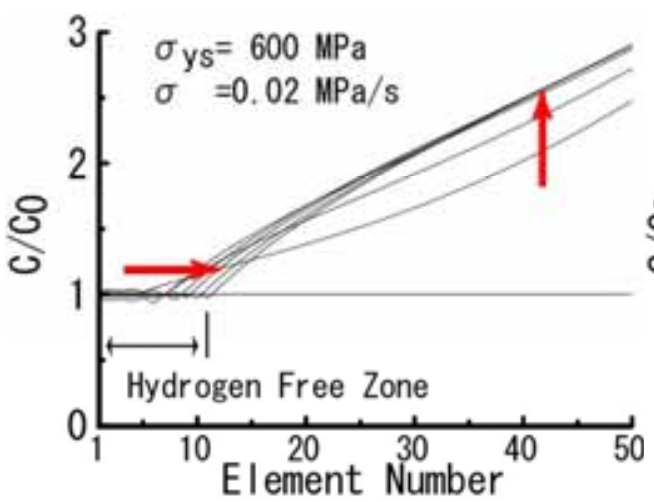

Fig. 7 Hydrogen distribution under low stress rate condition for a smooth specimen

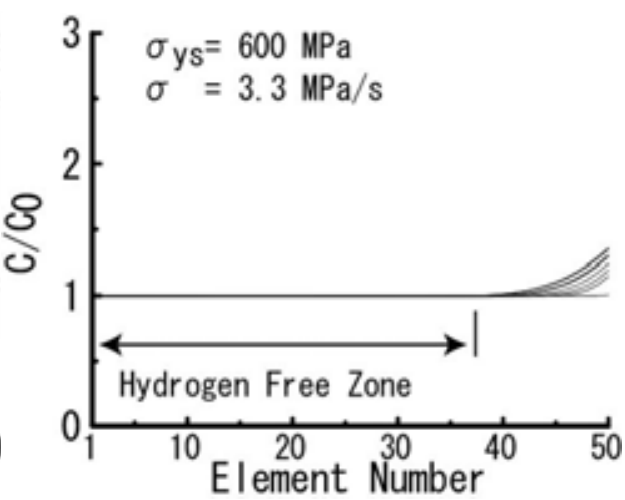

Fig. 8 Hydrogen distribution under high stress rate condition for a cracked specimen

In this case, the distance between a crack tip and the tip of hydrogen distribution, that is the region without hydrogen defined by hydrogen free zone is very small.

Under high stress rate condition, on the other hand, hydrogen penetration from outer surface is not so remarkable and large hydrogen free zone exists. Experimental results of hydrogen embrittlement for smooth specimen show that a trigger point is a non metallic inclusion and quasi cleavage fracture at first occurs from a trigger point accompanied by intergranular fracture due to hydrogen embrittlement. [13] However, under low stress rate condition, intergranular cracking takes major area of whole fracture surface. [13] In the numerical results of this paper, if quasi cleavage and intergranular fracture occur in the hydrogen free zone and in the region of hydrogen distribution respectively, this numerical result well predict the experimental results of hydrogen embrittlement for a smooth specimen. [13] Furthermore, the behavior of hydrogen embrittlement for a smooth specimen was also found to be dominated by applied stress rate as well as that for a cracked specimen as is shown in $\S 3.1$. The fracture mechanism of hydrogen embrittlement for a smooth specimen was illustrated as shown in Fig.9.

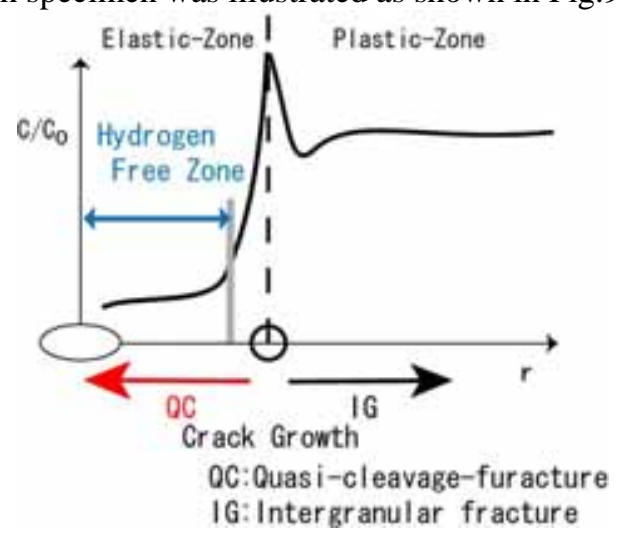

Fig. 9 The fracture mechanism of hydrogen embrittlement for a smooth specimen 


\section{CONCLUSION}

The analyses of hydrogen diffusion and concentration behavior under hydrogen atmospheric condition were conducted and the following conclusions were obtained.

1) The behavior of hydrogen embrittlement for a cracked specimen is dominated by applied stress rate. Low stress rate application causes typical hydrogen embrittlement due to the wide distribution of hydrogen, which results in the decrease in fracture toughness. On the other hand, high stress rate application causes localization of hydrogen embrittlement around a crack tip due to localization of hydrogen distribution around a crack tip.

2) For a smooth specimen, hydrogen free zone exists between a crack tip and the tip of hydrogen distribution, where quasi cleavage fracture occurs. Under low stress rate condition, hydrogen free zone is very small and hydrogen well penetrates from outer surface which results in wide area of intergranular fracture due to hydrogen embrittlement. On the other hand, under high stress rate condition, hydrogen free zone becomes large, typical quasi cleavage fracture accompanied by interguanular fracture can be clearly observed.

3) Concussions in 1) and 2) are well predicted by numerical analyses of hydrogen diffusion and concentration and those behaviors are sensitive to applied stress rate.

\section{Acknowledgements}

This work was partially supported by the Grant-in-Aid for 21 th Century COE program (The Exploration of the Frontiers of Mechanical Science Based on Nanotechnology), The Ministry of Education, Culture, Sports, Science and Technology is gratefully acknowledged.

2. Y. Wada, Proc. of the 2003 Annual Meeting of the JSME/MMD, 795, 2003

3. Y, Wada, T. Hasegawa and Y. Inoue, Proc. of the 49th Japan National Symposium on strength, Fracture and Fatigue, 71, 2004, in Japanese

4. A. T. Yokobori Jr., T. Uesugi, M. Sendoh and M. Shibata, Strength, Fracture and Complexity An International Journal, 1, 4, 2003 in press

5. A. T. Yokobori Jr., T. Nemoto, K. Satoh, T. Yamada, Engng. Fract. Mech., 55, 47, 1996

6. A. T. Yokobori Jr., Y. Chinda, T. Nemoto, K. Satoh, T. Yamada, Corrosion Science 44, 407-424, 2002

7. A. R. Troiano, J. Iron Steel Inst., 189,5,37, 1958

8. R.A Oriani, Hydrogen in metals, In Fundamental Aspects of Stress Corrosion Cracking, NACE 32, 1969, R.W. Staehle, A.J. Forty, D. Van Rooyen Eds., NACE, Houston

9. H.P. Leeuwen, Engng. Fracture. Mechanics. 6, 141, 1974

10. H.P. Leeuwen, Corrosion -NACE, 31, 2,42, 1975

11. M. Creager and P. C. Paris, Int. J. Fracture Mech., 3,247, 1967

12. R.Hill, The Mathematical Theory of Plasticity, Oxford University Press, Oxford, 242, 1954

13. M. Ishikawa and K. Yamada, Trans. of the Japan Soc. of Mech. Engineers, 59, 57, 1993

14. H. Yatabe, K, Yamada, E, R, DE Los Rios and K, J, Miller, Fatigue Fract. Engng. Struct. 18, 3, 377, 1995 